\title{
Improving Evaluation to Address the Unintended Consequences of Health Information Technology:
}

\section{a Position Paper from the Working Group on Technology Assessment \& Quality Development}

\author{
F. Magrabi ', E. Ammenwerth ${ }^{2}$, H. Hyppönen ${ }^{3}$, N. de Keizer ${ }^{4}$, P. Nykänen ${ }^{5}$, M. Rigby ${ }^{6}$, P. Scott ${ }^{7}$, \\ J. Talmon ${ }^{8}$, A. Georgiou' \\ 1 Australian Institute of Health Innovation, Macquarie University, Sydney, Australia \\ 2 UMIT, University for Health Sciences, Medical Informatics and Technology, Hall in Tyrol, Austria \\ ${ }^{3}$ National Institute for Health and Welfare, Information Department, Helsinki, Finland \\ ${ }^{4}$ Academic Medical Center, Department of Medical Informatics, Amsterdam, The Netherlands \\ ${ }^{5}$ University of Tampere, School of Information Sciences, eHealth Research, Finland \\ ${ }^{6}$ Keele University, School of Social Science and Public Policy, Keele, United Kingdom \\ 7 School of Computing, University of Portsmouth, Portsmouth, United Kingdom \\ 8 Hi-Way, Sint Odiliënberg, The Netherlands
}

\begin{abstract}
Summary
Background and objectives: With growing use of IT by

healthcare professionals and patients, the opportunity for any

unintended effects of technology to disrupt care health processes

and outcomes is intensified. The objectives of this position paper

by the IMIA Working Group (WG) on Technology Assessment and

Quality Development are to highlight how our ongoing initiatives

to enhance evaluation are also addressing the unintended conse-

quences of health IT.

Methods: Review of WG initiatives

Results: We argue that an evidence-based approach underinned by rigorous evaluation is fundamental to the safe and effective use of

IT, and for detecting and addressing its unintended consequences in a timely manner. We provide an overview of our ongoing initiatives to strengthen study design, execution and reporting by using evaluation frameworks and guidelines which can enable better characterization and monitoring of unintended consequences, including the Good Evaluation Practice Guideline in Health Informatics (GEP-HI) and the Statement on Reporting of Evaluation Studies in Health
\end{abstract}

Informatics (STARE-HI). Indicators to benchmark the adoption and impact of IT can similarly be used to monitor unintended effects on healthcare structures, processes and outcome. We have also developed EvalDB, a web-based database of evaluation studies to promulgate evidence about unintended effects and are developing the content for courses to improve training in health IT evaluation. Conclusion: Evaluation is an essential ingredient for the effective use of IT to improve healthcare quality and patient safety. WG resources and skills development initiatives can facilitate a proactive and evidence-based approach to detecting and addressing the unintended effects of health IT.

\section{Keywords}

Medical informatics; evaluation studies; evidence-based medicine; information systems; patient safety

Yearb Med Inform 2016:61-9

http://dx.doi.org/10.15265/IY-2016-013

Published online November 10, 2016

\section{Introduction}

All health information technology (IT) affects patients - some directly, such as decision support systems; some as a core part of modern clinical processes, such as electronic health records (EHR), ordering and prescribing; and others in the organisation of care such as scheduling and recall systems. As with all health interventions, and as with all informatics systems, these technologies can work well as intended, or they can fall short of intention; and somewhat regardless of their success against design they can have unanticipated negative consequences or unintended effects [1].

Reporting and discussion about the unintended consequences of health IT dramatically increased following Ash, Berg and Coiera's 2004 paper [2]. While this paper specifically focused on the consequences of errors at the interface of IT and work practice, subsequent studies have examined a range of unintended consequences associated with the different types of IT [1, 3-5]. For example, Campbell and colleagues reported nine categories of issues associated with order entry systems [6]. More recently, the focus has shifted towards discussing strategies to address these issues particularly in context of the large-scale implementations that are underway in many countries [7]. With rapidly growing use of IT the opportunity for unintended effects is intensified [8]. For instance, in the USA, 94\% of hospitals used EHRs in 2014, a fivefold increase since 2008. Indeed unintended effects due to poor integration with clinical workflow were identified as a top technology issue for US hospitals in 2016 [9].

Unintended effects not only disrupt the delivery of care but also pose risks to patient safety $[10,11]$. The adverse outcomes against design intention may be due to context factors such as different health settings, different clinical domains, technical configuration context, or users not adequately prepared [12]. The wider unintended consequences can include staff dissatisfaction (even to the point of seeking exit from that work setting), unofficial and risky work practices such as 'workarounds' to avoid interfacing with the computer system, changes to workflow of 
other departments, disruption of collaborative working in the health care sociotechnical environment, disruption of quality assurance and audit processes, and new costs incurred even though savings may be made elsewhere in the health ecosystem [13]. In extremis these unintended consequences can include iatrogenic effects or death [2, 14-16]. More frequently, achieved benefits can be partially nullified by unintended consequences - for instance an efficiency through an overall decrease in tests ordered because of better retrieval of earlier results may be offset by inefficient staff access to the system.

The IMIA Working Group (WG) on Technology Assessment and Quality Development seeks to address the unintended consequences of health IT as part of its broader initiatives to enhance evaluation. Our objectives in this position paper are to highlight the role of WG initiatives to address unintended consequences. In doing so we will show that evaluation is central to identifying and remediating unintended consequences when IT is implemented in complex sociotechnical organizations. We begin with an overview of the WG's mission and aims. In section 3 we describe our efforts to promote an evidence-based approach to evaluation. Initiatives to strengthen study design and execution so that unintended effects can be reliably measured are reviewed in section 4. Section 5 examines guidelines to enhance the reporting of evaluation studies, and the final section focuses on resources and skills development. The underlying rationale is to facilitate a proactive and evidence-based approach and continuously learn through timely and effective evaluations, and promulgation of findings.
- Develop and promote methods and tools to support the systematic evaluation of the effects of health IT on structure, process and outcome of patient care, and to give feedback to system builders on how to alter their systems to improve effectiveness and to avoid negative effects.

We are convinced that health IT evaluation demands a combined, multi-disciplinary as well as multi-method approach. Therefore, the aims of our WG are to:

a. Foster discussion between experts from informatics, medical informatics, economics, health care, health services research, clinical epidemiology, biometry, psychology, sociology, ethnography, organizational development, operations research and other evaluation fields, on an international level, and to encourage exchange on methodological issues between researchers from different traditions;

b. Provide opportunities to share knowledge with the aim of obtaining profitable cross-fertilization among different fields of expertise and especially between quantitative and qualitative research;

c. Promote a combined research agenda to develop frameworks and toolkits for information systems evaluation, offering guidelines for an adequate combination of evaluation methods and tools;

d. Discuss and clarify the networking needs for long-term evaluation research in medical informatics, and to promote combined research proposals at an international level.

\section{Promoting an Evidence- based Approach to Health IT}

An evidence-based approach to system design, implementation and use is fundamental to minimising unintended effects of health IT. For almost 12 years our WG has played a central role in coordinating the drive to develop and promote Evidence Based Health Informatics (EBHI) [17]. This cross-disciplinary movement was initiated by Ammenwerth [18], and developed into a cause that was taken up by IMIA as the theme for its 2013 Yearbook. It takes the fundamental stance that health informatics, like any other health science, should be based on evidence and that its interventions should be proved in practice as effective and safe by harnessing rigorous impartial evaluation [18]. This should be seen as part of an open and transparent evaluation imperative involving all stakeholders (IT vendors, health care professionals and healthcare commissioners) to ensure safety and quality of health IT.

The surprise might be that it has taken so long to raise EBHI systematically, some 60 years after the first computer applications in health. As long ago as 1991 Wall was arguing that vendor claims should be verified by scientific evaluation processes [19]. Three reasons for this delay might be postulated - that IT investment policy and decisions are frequently taken in economic and management contexts, even though they affect patients and practitioners, and they usually come under management rather than health professional control; secondly, that the ubiquity of IT applications in commercial and social activities disguises the fact that in healthcare they have a direct impact on patients, clinical processes and health outcomes; and thirdly that half a century of industry-driven processes have led to these being seen as the normal driving force, rather than health IT being the commissioned agent of healthcare delivery professionals and processes. But whatever the reasons, given the centrality to clinical and health care delivery processes, and the adverse outcomes of unintended consequences in terms of resource waste, delay, and on occasions death, our WG is committed to promoting further scientific development and application in this field.

\section{Strengthening the Design and Execution of Evaluation Studies}

Just as any other research discipline, the health informatics profession needs to be rigorous in its attempts to ensure the highest quality of research evidence. It is now widely recognized that a range of different quantitative, qualitative and mixed methods need to 
be used to assess the effects of health IT. Thus rigorous in this context means using appropriate methods to answer evaluation questions at hand. To this end, our WG actively promotes the use of evaluation frameworks to systematically examine the effects of health IT. We have developed guidelines and indicators as tools to enhance the quality of evaluation studies [20]. As will be shown in this section these initiatives are also likely to lead to better detection of unintended consequences.

\subsection{Evaluation Frameworks}

There are several frameworks that appear particularly relevant when considering the identification and assessment of unintended consequences [21]. We can distinguish between philosophical, conceptual and methodological frameworks.

- Philosophical frameworks attempt to propose foundational principles to underpin our apprehension and comprehension of a problem. Dooyewerd's fifteen "aspects" have been proposed as a unifying philosophical framework for understanding information systems [22]. As examples, the "lingual" aspect might be used to explore whether users correctly understand what a screen layout is telling them; or the "juridical" aspect might inform examination of an application's compliance with regulations or standards.

Taking a different approach House proposed that evaluation should be based on the principles of truth, coherence ('beauty') and justice [23]. He described eight basic categories of evaluation, upon which Friedman \& Wyatt based their health informatics study typology [24]. By analogy, these three basic principles have obvious application to health IT that make questionable benefit claims, may not integrate well or perhaps create inequities in workload impact.

- Conceptual frameworks provide a descriptive ontology to articulate and reason upon the phenomena under investigation, and might either be hypothetical or validated by research evidence. General information systems approaches such as the DeLone \& McLean success model [25], and the unified theory of accep- tance and use of technology (UTAUT) [26] provide a "language" for describing and decomposing health IT evaluations. When studying unintended consequences, component dimensions of these frameworks with particular importance are the information quality, system quality, service quality and user satisfaction elements of the DeLone \& McLean model and the aspects of performance expectancy, effort expectancy, social influence and facilitating conditions in UTAUT.

The FITT framework ("Fit between Individuals, Tasks and Technology") is another example that focuses on the human-computer interface, it proposes that clinical technology adoption depends on how well each of its three elements fit together [27]. This model quite obviously lends itself to understanding unintended consequences, given the typical usability and workflow consequences that can arise from health IT implementations.

Taking a broader view Ash and colleagues developed a Thematic Hierarchical Network Model of unintended consequences based on their extensive research of order entry systems [28]. The nine types of consequences for order entry offer transferable lessons for other forms of health IT intervention. Another framework that was specifically derived to address unintended consequences is the ISTA ("Interactive Sociotechnical Analysis") [29]. ISTA posits five emergent and recursive interactions between infrastructure, social context, new health IT and established health IT that constitute a sociotechnical system with the potential to go wrong in unforeseen ways.

- Methodological frameworks go farther, specifying or suggesting the steps and techniques to employ in the evaluation. The AHRQ health IT toolkit is fairly abstract and does not directly address unintended consequences, but does highlight the need to use both qualitative and quantitative methods to obtain a comprehensive understanding [30]. Its proposed structure for an evaluation plan recommends defining questions to explore both positive and negative impacts of the intervention.
A more systematic approach is provided by realist evaluation which asks "what works for whom, in what circumstances and in what respects, and how?' [31]. Realist evaluation emphasises theory and explanation, use of multiple methods (but with considerable scepticism about experimental designs for programme theories) and aims to understand how the context, mechanism(s) of action and outcomes (C-M-O) interrelate. Theory of Change is another way to describe and break down how 'change' is anticipated to work in a given programme [32]. It starts from the long-term goals and works backwards to map the required pre-conditions, causal chains, interventions, assumptions, rationale and indicators. It considers the context, actors; the product is "a working model against which to test hypotheses and assumptions about what actions will best bring about the intended outcomes" [33]. Both of these frameworks are clearly applicable to analysis of unintended consequences, as they explore how and why interventions work or not.

\subsection{Guideline for Good Evaluation Practice in Health Informatics}

The Good Evaluation Practice Guideline in Health Informatics (GEP-HI) supports planning and execution of a health IT evaluation study [34]. GEP-HI was based on existing knowledge, experience and literature on evaluation studies, methodologies, guidelines development, codes of ethics and good implementation practices e.g. $[24,35,36]$. The guideline was developed through an informal consensus-seeking process, without balloting, in the community of health IT evaluation experts, and it has been regularly in open discussion through the HISEVAL website (http://iig. umit.at/efmi) and many conference workshops.

The objective of the GEP-HI is to give advice on how to design and carry out evaluation studies in various health IT contexts. The guideline lists issues to consider, and gives recommendations on how to design evaluation studies, how to make methodological choices, how to conduct studies and how to define evaluation criteria at specific phases of the health IT life cycle. 
GEP-HI is divided into parts corresponding to the phases of an evaluation study and the theoretical background for the study phases is compliant with models for information system development [34].

\section{GEP-HI distinguishes six phases:}

1. Preliminary outline presenting the purpose of the study and the first ideas on why, for whom, and how the evaluation should take place,

2. Study design clarifying the design issues for the evaluation study,

3. Operationalization of methods making the methodological approach and methods concrete and compliant with the system type, the organization and the information need,

4. Project planning developing plans and procedures for the evaluation project,

5. Execution of the evaluation study accomplishing the designed evaluation study,

6. Completion of the evaluation study reporting, accounting, archiving of evaluation study results, finalization of outstanding issues and formal closure of the evaluation study.

All phases together contain some 60 detailed items, which are presented in relation to the evaluation study phases [34]. GEP-HI can be applied to different kinds of health IT evaluation studies, irrespective of whether the object of study is an IT application or a method like nursing classification or data security practice. When designers and executers of evaluation studies address these items, the plan, structure, objectives and results of the studies will become more robust and consequently the studies contribute an important step towards evidence-based health informatics. When applied, GEP-HI has potential to raise the quality of evaluation studies through careful planning, and thus contribute to the accumulation of the scientific evidence base.

\subsection{Development of E-health Indicators}

Rapid technological diffusion has created a need to benchmark the adoption and impact of health IT, to learn from initiatives, and allow managers and other decision makers to make informed decisions about their systems. To this end, our WG is fostering the development of internationally compatible indicators to examine the impact of IT on key dimensions of healthcare system performance - structures, processes and outcomes [18].

Indicators that focus on measuring the effects of IT on healthcare structures (e.g. system and information quality) are associated with impacts on processes (e.g. system use and user satisfaction) and outcomes. Unintended structural impacts have consequences on processes, and this in turn affects outcomes [25]. The consequences become apparent when indicators are compared to target values in different contexts of use. However, the outcomes can often be attributed to a wide range of factors: many factors beyond EHR impact, for example patient safety. In this section we present some examples of unintended impacts and consequences that can be captured by monitoring change in performance indicators for health IT, mainly defined in the context of the OECD and Nordic collaboration [37]

Examples of indicators to monitor unintended effects on healthcare structures

- Delayed implementation: e.g. "Proportion of public organisations (hospitals/ health centres/ private clinics) where a list of prescriptions made to the patient outside own organisation (nationally) is available for professionals". In Finland, this indicator has been monitored in real-time by organisation and vendor system to indicate deviations from targets set by the e-health legislation during implementation of the national e-prescription system.

- Exceeding the budget: A national level indicator used for Nordic benchmarking is "IT costs as a proportion of total budget". These data are collected with national organisational surveys, making it possible to benchmark IT costs of different organisations with different vendor systems in relation to implementation of a range of IT functionalities.

- Unreliability of system post-implementation: System stability is a prerequisite for use. An indicator "mean satisfaction experienced by clinicians with system reliability" has been used to monitor stability of the system. Actual downtime is a more objective indicator, but data for that is not readily available. Data were collected from national physician surveys, and reported nationally per context and vendor system. For example, data from Finland showed a slight decrease in overall stability from 2010 to 2014, while Iceland showed slightly lower overall scores compared to Finland.

- Negative user emotions: This indicator has been monitored in the Nordic countries using "user satisfaction score". Overall satisfaction was relatively low in 2014, has decreased somewhat in Finnish primary care, increased in specialist care, and was slightly better in Iceland compared to Finland.

Examples of indicators to monitor unintended effects on healthcare processes:

- Conflicting priorities: For example, regulatory compliance versus clinical workflow. In the Finnish physician survey, scores for the statement "compiling statistics on patient information takes too much time i.e. documenting information needed for billing, national registries etc." were very poor for the majority of vendor systems [38].

- Conflicts with the workflow: Poor scores were recorded for the commonly defined Nordic indicators including "health IT support for routine tasks", "time required for patient data retrieval and documentation", "changes in communication patterns and practices". These may all have contributed to non-use of the system, which was monitored from $\log$ data by "Frequency of use of prescription data from outside own organisation per population via national or regional system". This indicator scored poorly in all other countries apart from Denmark.

- From follow-up monitoring data, it is also possible to infer unintended process impacts like generation of new kinds of errors, reduced learnability and vendor reluctance to correct errors. Finnish physicians reported that: "information 
entered/documented occasionally disappears from the information system", "learning to use the EHR does not require a lot of training" and "suggested corrections and changes are performed sufficiently rapidly" [39].

Unintended effects on healthcare outcomes such as adverse impact on patient safety have been monitored in the Nordic countries by the indicator "IT system has caused or nearly caused an adverse event". The mean score for the Nordic countries was above 3 (scale 1-5, where 1=high, $5=$ low) with Finland scoring best. Patient data is increasingly collated from different provider systems to national databases, and if there are problems with data integrity, consistency, availability or data protection, patient safety may be directly or indirectly compromised. Systematic monitoring of information security-related patient data quality would require access to patient data in the national databases, which is to a large extent still not possible.

Similarly, other lower than expected values for outcome indicators can be used to monitor unintended impacts of health IT. For example in Finland, a national mental health portal intends to reduce number of face-to-face-visits with mental health problems. This impact is currently being monitored from the national statistics by comparing number of visits per client in regions of low usage with regions of high usage. Portal usage rate is obtained using Google analytics.

In future, much of the health IT implementation stage, data quality, usage rate and impact information could be obtained from the national health information systems, if the system log and patient information were available for monitoring. This would make it possible to develop automatic online monitoring systems of key indicators. This, in turn, would allow for immediate actions where deviations occur. A prerequisite for this type of system is that the logs are specified to allow extraction of required usage data, and the legislation allows patient information use for secondary purposes. The Nordic network plan for period 2015-2017 is to pilot this type of system with information that is currently available.

\section{Improving the Quality of Reporting about the Effects of Health IT}

It is essential to have access to published evaluation studies to be able to evaluate positive effects but also unintended consequences of health IT. Published studies should contain the necessary information to understand approach, methods and results of an evaluation study, and to judge its strengths, weaknesses, sources of bias and especially the generalizability of its results. Incomplete or unclear study publications can lead to misinterpretation and hamper the evidence-base of health informatics. However, authors of systematic reviews often report many problems with the publication quality of health IT evaluation studies.

To address this concern, the STARE-HI guidelines - Statement on Reporting of Evaluation Studies in Health Informatics - were developed and published in 2009 [40]. The STARE-HI checklist comprises 30 items which should be considered to be included in a publication of an evaluation study (see Table 1). The arguments for each item as well as good and bad examples have been published in an explanation and elaboration paper, which is available as an open access publication [41]. For conference papers not all items can be addressed, therefore a prioritization of the STARE-HI items has been published particularly for that purpose [42]. Alongside IMIA, STARE-HI has been endorsed by major health informatics journals and the European Federation for Medical Informatics (EFMI). It has also been included in the EQUATOR network [43]. Further details about STARE-HI are

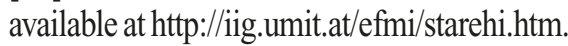

Unintended consequences have - by definition - not been expected and are thus typically not included in the prospectively planned evaluation criteria of an evaluation study. STARE-HI nevertheless includes ways to report on identified unintended consequences of health IT: in section 7.4 of the STARE-HI principles, any unintended (positive or negative) side-effects of the evaluated systems should be reported (see Table 1). These unexpected observations can surface both in quantitative and qualitative studies. STARE-HI proposes to report these unexpected observations, and to discuss possible reasons and implications, as this information may help provide new insights of the impact of the health IT system. Unintended consequences may not only be related to the health IT system itself, but also to the way it has been introduced into the organization, including aspects such as changes in internal hospital policies or workflows.

We expect that STARE-HI may help to improve the publication quality of health IT evaluation papers. This in turn would strengthen the evidence-base of health informatics and allow others to identify both expected and unintended consequences based on high-quality health IT evaluation publications.

Table 1 The STARE-HI principles: Items recommended to be included in health IT evaluation reports [40].

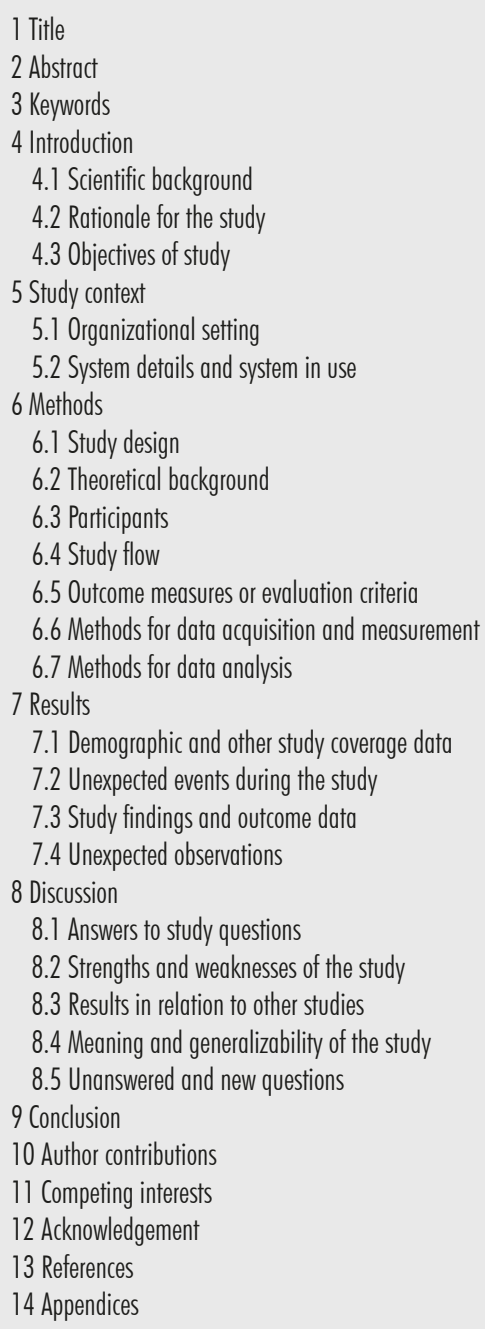




\section{Supporting Resources and Developing Skills}

In this penultimate section we describe our initiatives to promulgate evidence about unintended effects and to improve training in health IT evaluation. Systematic evaluation studies are needed to verify that appropriate benefits are forthcoming and no unintended side effects of health IT are obtained [17]. To ensure that these evaluations are conducted in accordance with appropriate scientific and professional standards, well-trained health informatics experts are needed.

\section{Web-based Health IT Evaluation Inventory}

Available keywords often do not cover the specifics of health IT evaluation studies, for example regarding type of evaluated health IT system, clinical context, applied evaluation methods, or used evaluation criteria. To overcome this challenge, we established the web-based evaluation database EvalDB in collaboration with the European Federation of Medical Informatics WG on "Evaluation in Health Information Systems" (https:// evaldb.umit.at)[44] [17].

At present EvalIDB contains more than 1,800 evaluation studies and systematic reviews of health information technology and is available free of charge. EvalDB was created to help researchers to identify studies that have been conducted. Information on the evaluation studies is presented using predefined categories offered for language of publication, country, type of information system, evaluation approach, type of research, clinical domain, study environment, and evaluation criteria [44]. In addition, the entries are linked to the related PubMed entry of the study publication.

EvalDB is based on systematic literature searches in PubMed, and it is updated from time to time. Researchers can also propose studies to be included in EvalDB by the same website. The challenge to develop such a repository is the completeness and the currency of its entries, as extracting and classifying the evaluation studies is time-consuming and based on voluntary work.
EvalDB at the moment does not include a structured description of unintended consequences of health IT in the event that they were detected in an evaluation study. Only if these unintended consequences are mentioned in the abstract in the paper, they are classified together with the other prospectively planned evaluation criteria. It would be an interesting add-on to EvalDB to classify unintended consequences separately. This would allow identifying evaluation studies that found certain types of unintended consequences and make further analysis easier (e.g. the relation between types of health IT and types of unintended consequences). This, however, will require the full-text analysis of all evaluation papers, as unintended consequences are typically not reported in abstracts.

Meanwhile, examples of unintended consequences of health IT have been collected in the form of case studies on a dedicated website maintained by our WG and available at https://iig.umit.at/efmi/badinformatics. $\mathrm{htm}$. The website collects descriptions of reported incidents with health IT. For each incident or problem at least one link to a source is provided. The website also contains a list of recommended further readings on unintended consequences of health IT.

\section{Courses on Health IT Evaluation}

The IMIA recommendations on health informatics education [45], state that "evaluation and assessment of information systems" should be part of health informatics curricula. These recommendations identify some exemplary content for such training but give no further details.

To address this gap, university lecturers were invited to provide structured descriptions of successfully running health IT evaluation courses. Overall, structured descriptions from ten courses from universities in Europe, United States and Australia were collected [46]. To develop the recommendations for health IT evaluation courses, input from these existing courses was combined with a series of workshops held during international Medical Informatics conferences. These activities were coordinated by an expert group from our
WG in collaboration with the health IT evaluation WGs of EFMI and the American Medical Informatics Association.

The general recommendations of the course are:

- Course objective: Introduce participants to theory and practice of health IT evaluation; at the end, the students should be able to plan an own (smaller) evaluation study, to select and apply selected evaluation methods, and to perform a study and report its results; also, they should be able to appraise the quality and the results of published health IT evaluation studies.

- Level of the course: Master or postgraduate level.

- Duration of the course: The mandatory core topics can be taught in a course of 6 ECTS (European Credit Transfer and Accumulation System), equivalent to 150 hours of overall student workload (both classroom time and homework). The duration of the course can be longer if facultative content or extended practical training is added.

- Participants: multidisciplinary groups of students, with backgrounds in computer science, health IT, medicine, nursing, social science, information sciences, or business.

- Practical training: including practical training focussing on different aspects of evaluation, depending on the learning objectives, the level of participants, and the available time.

- Prerequisites: Before joining the health IT evaluation course, the students should have obtained knowledge in the following areas (these areas are thus not included in the recommendations):

- Philosophy of science

- Scientific evidence

- Designing a research study

- Research ethics

- Quantitative research methods and statistics

- Qualitative research methods

- Project management

The recommended core content, split into mandatory and facultative content, is currently under review by the core team but preliminary results are listed in Table 2 [18]. 
The recommendations assume that all listed items are taught on an introductory level which includes examination of unintended effects. Extended expert knowledge e.g. in qualitative evaluation methods could then be covered in subsequent specialized lectures. Also the listed facultative topics could also be taught in dedicated specialized lectures after a core module on health IT evaluation.

\section{Challenges and Limitations in the Generation, Promotion and Application of Evidence- based Research Findings}

The existence of unintended consequences associated with health IT, has led to concern about their potential to inhibit the full realisation of the benefits of IT, and their capacity to contribute to diagnostic errors [47]. This position paper has established that IT (like any other technology involving patient care) needs to be designed, implemented and monitored carefully [12]. Evaluation performs a central role in identifying and remediating the unintended consequences of health IT and is a critical component of an evidence-based (i.e. EBHI) approach to providing the best available evidence about effective and safe use of IT in practice [17].

Early detection and open reporting of unintended consequences of health IT systems and infrastructures are fundamentally important for a number of reasons. The first and obvious one is that any event that adversely affects patients directly such as corrupted or missing data, or compromised processes, cuts fundamentally against the core purpose of the health system. Secondly, unintended outcomes that reduce efficiency or effectiveness of an aspect of a health system similarly degrade the system's support of patients. Open reporting and investigation are also important, as time spent overcoming denial of such effects (whether by vendors or organisational management) simply wastes further time. Finally, lack of reassurance or trust in a system will adversely affect users' (especially health professional users') effective use of

Table 2 Preliminary recommendations for mandatory and facultative content for health IT evaluation courses [18].

\begin{tabular}{|l|l|}
\hline \multicolumn{2}{|c|}{ A Mandatory core topics } \\
\hline A1 & Need for evidence-based health informatics (i.e. health IT and patient safety) \\
A2 & Theories of evaluation (e.g. objectivist and subjectivist, formative and summative) \\
A3 & How to build an evaluation study (e.g. stakeholder analysis, tailor the evaluation, steps of an evaluation study) \\
A4 & Study designs for health IT evaluation studies (e.g. experimental, quasi-experimental, observational) \\
A5 & Indicators for health IT quality (structure, process, outcome quality) and their relation to clinical indicators \\
A6 & Measurement principles (e.g. objectivity, reliability, validity of measurements, types of bias) \\
A7 & Quantitative data collection methods in health IT evaluation \\
A8 & Qualitative data collection methods in health IT evaluation \\
A9 & Multi-methods approaches and triangulation \\
A10 & Quality of health IT evaluation studies \\
A11 & Reporting and publishing of an evaluation study \\
A12 & Finding a published evaluation study \\
A13 & Answer to "so, what..." questions - what do the evaluation results mean for IT management and clinical practice? \\
A14 & Practical training in health IT evaluation (e.g. write an evaluation plan based on a realistic case study; conduct a \\
& real evaluation project; criticize a published evaluation study) \\
\hline B Facultative topics (to be chosen based on available time and background of participants) \\
\hline B1 & Evaluation frameworks for health IT evaluation \\
B2 & Evaluation of user and technology acceptance \\
B3 & Evaluation of usability \\
B4 & Technical evaluation (software testing) \\
B5 & Evaluation of people and organizational impact \\
B6 & Evaluation of clinical impact \\
B7 & Economic evaluation \\
\hline
\end{tabular}

local health IT systems and thus exacerbate IT-induced inefficiencies, or needless unconstructive workarounds.

We have highlighted key components involved in the practice of EBHI beginning with the utilisation of appropriate frameworks, such as those designed to: a) assist in the methodological approach to evaluation (e.g., the steps and techniques necessary to undertake rigorous evaluations which includes choosing appropriate methods [48]; b) provide a conceptual framework to understanding the IT intervention under consideration (e.g., the usability of the system); c) establish the foundational or philosophical underpinnings of the evaluation (e.g., community, organisational and/or individual approaches). These frameworks can be supplemented by guidelines such as the GEP-HI and the STARE-HI which provide systematic approaches to the design and execution of evaluation studies and the generation of robust health informatics evidence. In addition, the EvalDB is a web-based inventory (with references and structured information) of evaluation studies in medical informatics EvalDB which was developed as a collaboration between the EFMI WG on "Evaluation in Health Information Systems" and the IMIA WG on "Technology Assessment and Quality Development."

A key part of health IT evaluation also involves the construction of reliable means of measuring its use, quality, safety and sustainability [12]. This includes the utilisation of performance indicators to monitor the functioning and effects of IT. Performance indicators can provide an important means of benchmarking IT safety, to provide comparisons with previous performance, across different systems or over time. 
Our recommendations need to be considered in light of some challenges and limitations of doing rigorous evaluation in practice. Firstly, there is a question of limited resources including budget allocated for evaluation post-implementation. Thus evaluation costs need to be proactively built into the overall budget. Secondly, evaluators need to recognise the very political nature of health IT implementations and the limitations that may be placed on them when undertaking and reporting studies especially unintended effects. Finally, the study of unintended effects tends to utilise qualitative methods to investigate low frequency events where it might not be possible to obtain representative samples to quantify effects. Indeed evaluators need to choose appropriate methods recognizing that representative samples are not required to characterise problems or to improve the safety and usability of health IT in a professional manner.

\section{Conclusion}

Health IT incorporates a broad range of systems and products, ranging from electronic health records, clinical information systems, and even health information exchanges. It is a dynamic area of development that involves technical systems (e.g., computers and software) within organisations that include people, processes and workflow [12]. IT systems support several processes ranging from the collection of data (e.g., clinical documentation), the communication of information (e.g., order entry and result reporting) and decision support (e.g. order sets, prompts and alerts), but increasingly IT systems drive key processes ranging from scheduling care aspects through to calculating their content and effecting their execution. In today's environment there is also a growing emphasis on IT systems which engage patients as partners in their own care [49]. There is a spectrum of features that contribute to the safe use of IT including their usability by people, their interoperability (exchange of health information between systems and across organisational boundaries) and their impact on workflows. In a safety-aware and outcome efficient health system patients and professionals need to trust that IT systems are both safe and effective, and this means that unintended consequences of such systems are sought rigorously and their elimination or control ensured. This is also core to evidence-based medicine principles, and so evidence-based health informatics and active seeking of evidence should be espoused universally. Evaluation needs to be a critical part of the design, implementation and sustainability of health IT, not as an afterthought but as an essential evidence-based ingredient to achieving safe and quality care. Evaluation can thus serve a purpose not only by informing decision making by commissioners but also in assisting developers of health IT about the specific economic requirements (e.g., return on investment) for technologies in development [50]. Seeking, and building on, this evidence should be an essential part of health IT implementation. Our Working Group's mission is to facilitate and encourage this process.

\section{References}

1. Ash JS, Sittig DF, Poon EG, Guappone K, Campbell E, Dykstra RH. The extent and importance of unintended consequences related to computerized provider order entry. J Am Med Inform Assoc 2007;14(4):415-23.

2. Ash JS, Berg M, Coiera E. Some unintended consequences of information technology in health care: the nature of patient care information system-related errors. J Am Med Inform Assoc 2004;11(2):104-12.

3. Weiner JP, Kfuri T, Chan K, Fowles JB. "e-Iatrogenesis": the most critical unintended consequence of CPOE and other HIT. J Am Med Inform Assoc 2007;14(3):387-8.

4. Rahadhan P, Poon SK, Land L. Understanding unintended consequences for EMR: a literature review. Stud Health Technol Inform 2012;178:192-8

5. Kuperman GJ, McGowan JJ. Potential unintended consequences of health information exchange. J Gen Intern Med 2013;28(12):1663-6.

6. Campbell EM, Sittig DF, Guappone KP, Dykstra RH, Ash JS. Overdependence on technology: an unintended adverse consequence of computerized provider order entry. AMIA Annu Symp Proc 2007:94-8.

7. Bloomrosen M, Starren J, Lorenzi NM, Ash JS, Patel VL, Shortliffe EH. Anticipating and addressing the unintended consequences of health IT and policy: a report from the AMIA 2009 Health Policy Meeting. J Am Med Inform Assoc 2011;18(1):82-90.

8. Coiera E, Aarts J, Kulikowski C. The dangerous decade. J Am Med Inform Assoc 2012;19(1):2-5.

9. ECRI Institute's 2016 Top 10 Health Technology Hazards. ECRI Institute, USA.

10. Magrabi F, Baker M, Sinha I, Ong MS, Harrison S, Kidd MR, et al. Clinical safety of England's national programme for IT: A retrospective analysis of all reported safety events 2005 to 2011 . Int J Med Inform 2015;84(3):198-206.

11. Magrabi F, Ong MS, Runciman W, Coiera E. Using FDA reports to inform a classification for health information technology safety problems. J Am Med Inform Assoc 2012;19(1):45-53.

12. Health IT and Patient Safety: Building Safer Systems for Better Care. Washington, DC: The National Academies Press: Institute of Medicine; 2012

13. Kuziemsky CE. Review of Social and Organizational Issues in Health Information Technology. Healthc Inform Res 2015;21(3):152-60.

14. Ammenwerth E, Shaw NT. Bad health informatics can kill--is evaluation the answer? Methods Inf Med 2005;44(1):1-3.

15. Han YY, Carcillo JA, Venkataraman ST, Clark RS, Watson RS, Nguyen TC, et al. Unexpected increased mortality after implementation of a commercially sold computerized physician order entry system. Pediatrics 2005;116(6):1506-12.

16. HI B. Bad Health Informatics can Kill [Available from: http://iig.umit.at/efmi.

17. Rigby M, Ammenwerth E, Beuscart-Zephir MC, Brender J, Hypponen H, Melia S, et al. Evidence Based Health Informatics: 10 Years of Efforts to Promote the Principle. Joint Contribution of IMIA WG EVAL and EFMI WG EVAL. Yearb Medical Inform 2013;8:34-46.

18. Ammenwerth E, Rigby M, editors. Evidence-Based Health Informatics - Promoting Safety and Efficiency through Scientific Methods and Ethical Policy. Amsterdam: IOS Press; 2016.

19. Wall R. Computer Rx: more harm than good? Journal Med Syst 1991;15(5-6):321-34.

20. Nykänen P, Kaipio J. Quality of health IT evaluations. In: Ammerwerth E, Rigby M, editors. Evidence-based health informatics. Stud Health Technol Inform 2016;222:291-303.

21. Yusof MM, Papazafeiropoulou A, Paul RJ, Stergioulas LK. Investigating evaluation frameworks for health information systems. Int J Med Inform 2008;77(6):377-85.

22. Basden A. Philosophical frameworks for understanding information systems. Hershey, PA, USA: IGI Global; 2008.

23. House ER. Origins of the Ideas in Evaluating with Validity. New Directions for Evaluation. 2014;2014(142):9-15.

24. Friedman CF, Wyatt J. Evaluation methods in medical informatics, 2 Ed. New York: Springer Verlag; 2006.

25. Delone WH, McLean ER. The DeLone and McLean Model of Information Systems Success: A Ten-Year Update. J Manage Inf Syst 2003;19(4):9-30.

26. Venkatesh V, Morris M, Davis G, Davis F. User acceptance of information technology: toward a unified view. MIS Quarterly 2003;27(3):425-78.

27. Ammenwerth E, Iller C, Mahler C. IT-adoption and the interaction of task, technology and individuals: 
a fit framework and a case study. BMC Med Inform Decis Mak 2006;6:3.

28. Ash JS, Sittig DF, Dykstra RH, Guappone K, Carpenter JD, Seshadri V. Categorizing the unintended sociotechnical consequences of computerized provider order entry. Int J Med Inform 2007;76(Suppl 1):21-7.

29. Harrison MI, Koppel R, Bar-Lev S. Unintended consequences of information technologies in health care - an interactive sociotechnical analysis. J Am Med Inform Assoc 2007;14(5):542-9.

30. Cusack C, Byrne C, Hook J, McGowan J, Poon E, Zafar A. Health Information Technology Evaluation Toolkit: 2009 Update. Rockville, MD: Agency for Healthcare Research and Quality; 2009 [updated June. Available from: https:// healthit.ahrq.gov/sites/default/files/docs/page/ health-information-technology-evaluation-toolkit-2009-update.pdf.

31. Pawson R, Manzano-Santaella A. A realist diagnostic workshop. Evaluation 2012;18(2):176-91.

32. De Silva MJ, Breuer E, Lee L, Asher L, Chowdhary N, Lund C, et al. Theory of Change: a theory-driven approach to enhance the Medical Research Council's framework for complex interventions. Trials 2014;15:267.

33. Taplin DH, Clark H, Collins E, Colby DC. Theory of Change technical papers New York: ActKnowledge; 2013 [updated 31 July 2015. Available from: http://www.theoryofchange.org/wp-content/ uploads/toco_library/pdf/ToC-Tech-Papers.pdf.

34. Nykanen P, Brender J, Talmon J, de Keizer N, Rigby $\mathrm{M}$, Beuscart-Zephir MC, et al. Guideline for good evaluation practice in health informatics (GEP-HI). Int J Med Inform 2011;80(12):815-27.

35. Brender J. Handbook of evaluation methods for health informatics. USA: Elsevier Academic Press; 2006.

36. Kaplan B, Shaw NT. Future directions in evaluation research: people, organizational, and social issues. Methods Inf Med 2004;43(3):215-31.

37. Hyppönen H, Kangas M, Reponen J, Nøhr C, Villumsen S, Koch S, et al. Nordic eHealth Benchmarking: Status 2014: Nordisk Ministerråd; 2015.

38. Lääveri T, Vainiomäki S, Kaipio J, Reponen J, Vänskä J, Lehtovirta M, et al. Yksityissektorin potilastietojärjestelmät arvioitu 2014 [Evaluation of private sector patient information systems 2014]. Finnish Medical Journal 2015;23:1660-7.

39. Hyppönen H, Hämäläinen P, Reponen J, editors. E-health and e-welfare of Finland. Check point 2015. Helsinki, Finland: National Institute for Health and Welfare; 2015.

40. Talmon J, Ammenwerth E, Brender J, de Keizer N, Nykanen P, Rigby M. STARE-HI--Statement on reporting of evaluation studies in Health Informatics. Int J Med Inform 2009;78(1):1-9.

41. Brender J, Talmon J, de Keizer N, Nykanen P, Rigby M, Ammenwerth E. STARE-HI - Statement on Reporting of Evaluation Studies in Health Informatics: explanation and elaboration. Appl Clin Inform 2013;4(3):331-58.

42. de Keizer NF, Talmon J, Ammenwerth E, Brender J, Rigby M, Nykanen P. Systematic prioritization of the STARE-HI reporting items. An application to short conference papers on health informatics evaluation. Methods Inf Med 2012;51(2):104-11.

43. EQUATOR. The Equator Network: Enhancing the Quality and Transparency of Health Research 2014 [Available from: http://www.equator-network.org.

44. Ammenwerth E, de Keizer N. An inventory of evaluation studies of information technology in health care trends in evaluation research 19822002. Methods Inf Med 2005;44(1):44-56.

45. Mantas J, Ammenwerth E, Demiris G, Hasman A, Haux R, Hersh W, et al. Recommendations of the International Medical Informatics Association (IMIA) on Education in Biomedical and Health
Informatics. First Revision. Methods Inf Med 2010;49(2):105-20.

46. Eval EW. Curricula of health IT evaluation courses 2014 [Available from: https://iig.umit.at/efmi/ curricula.htm

47. Balogh E, Miller B, Ball J. Improving Diagnosis in Health Care. Institute of Medicine. National Academies Press; 2015.

48. Poon EG, Cusack CM, McGowan JJ. Evaluating healthcare information technology outside of academia: observations from the national resource center for healthcare information technology at the Agency for Healthcare Research and Quality. J Am Med Inform Assoc 2009;16(5):631-6.

49. Rigby M, Georgiou A, Hypponen H, Ammenwerth E, de Keizer N, Magrabi F, et al. Patient Portals as a Means of Information and Communication Technology Support to Patient- Centric Care Coordination - the Missing Evidence and the Challenges of Evaluation. A joint contribution of IMIA WG EVAL and EFMI WG EVAL. Yearb Med Inform 2015;10(1):148-59.

50. Ijzerman MJ, Steuten LM. Early assessment of medical technologies to inform product development and market access: a review of methods and applications. Appl Health Econ Health Policy 2011;9(5):331-47.

\section{Correspondence to:}

Associate Prof. Farah Magrabi

Centre for Health Informatics

Australian Institute of Health Innovation

Macquarie University

Sydney, NSW 2109

Phone: + 61298502429

Fax: +61 280886234

E-mail: farah.magrabi@mq.edu.au 